ORIGINAL ARTICLE

\title{
Serum Endocan Levels in Patients with Psoriasis Vulgaris as a Marker of Disease Severity
}

\author{
Ibrahim Elgarib ${ }^{1}$, Naglaa Khalifa ${ }^{1}$, Ola Elasser ${ }^{1}$,Mohamed ElGhareeb ${ }^{1}$, Doaa Hosny ${ }^{1}$ * \\ ${ }^{1}$ department of Dermatology, Venereology \& Andrology, Faculty of Medicine, Zagazig University, Egypt.
}

*Corresponding author:

Doaa Hosny Afara

Assistant lecturer of

Dermatology, Venereology \&

Andrology, Faculty of

Medicine, Zagazig University,

Egypt

Email:

doaahmeddodo84@gmail.com

\begin{tabular}{|l|l|}
\hline Submit Date & $2019-06-24$ \\
\hline Accept Date & $2019-07-14$ \\
\hline
\end{tabular}

\begin{abstract}
Background: Endocan is a specific molecule of human endothelial cell. It was accepted as a marker of endothelial dysfunction that is widely involved in psoriasis development. Our aim was to evaluate the relation between serum endocan levels and severity of psoriasis vulgaris . Methods: Twenty-two patients with psoriasis vulgaris were involved. We measured serum endocan, c-reactive protein (CRP) and erythrocyte sedimentation rate (ESR). Psoriasis severity was evaluated by Psoriasis Area and Severity Index (PASI) score. Results: The mean serum level of endocan was $154 \pm 49.3 \mathrm{pg} / \mathrm{ml}$ with a range from 59 to $210 \mathrm{pg} / \mathrm{ml}$. Its level was statistically significant increase in moderate and severe psoriatic patients compared to mild psoriatic patients. No statistically significant correlations were detected between serum endocan and CRP $(\mathrm{P}=0.11)$ and $\operatorname{ESR}(\mathrm{P}=0.16)$. In contrast, a very highly statistically significant positive correlation was detected between endocan and disease severity $(\mathrm{p}=0.001)$. Conclusions: Serum endocan can be a promising marker that correlates with psoriasis vulgaris severity. Key words: Endocan; psoriasis; endothelial dysfunction.
\end{abstract}

\section{INTRODUCTION}

$\mathrm{E}$ ndocan is a circulating proteoglycan that released from the vascular endothelium, previously termed endothelial cell specific molecule-1(ESM-1)[1].

The average concentration of endocan in serum of healthy individuals is $1 \mathrm{ng} / \mathrm{ml}[2]$. Increased expression of endocan in serum and tissue reflects neovascularization and endothelial activation that are major pathophysiological features associated with inflammation[3].

Psoriasis is a chronic immune-mediated inflammatory disease. It affects $2-3 \%$ of the people worldwide[4]. The cause of psoriasis is still unknown, however there is a complex interaction between genetic, environmental and immunological factors[5].

Psoriasis is characterized by hyperproliferation of the epidermis, augmented antigen presentation, production of T-helper 1 cytokines, expression of T-cell and angiogenesis[6].

Our study aimed to assess the relation between serum levels endocan and psoriasis vulgaris severity to detect if endocan could be used as a marker of severity in psoriatic patients.

\section{METHODS}

This cross sectional study included twenty-two patients with psoriasis vulgaris selected from the outpatient clinic of the Dermatology, Venereology \& Andrology Department, Zagazig University Hospitals during the period from September 2017 to September 2018.

The Institutional Review Board (IRB) at Faculty of Medicine, Zagazig University approved this study. All patients assigned a written informed consent. This work was conducted in accordance with The Code of Ethics of the World Medical Association 
(Declaration of Helsinki) for studies involving humans.

\section{Inclusion criteria:}

- Psoriasis vulgaris patients of any age and both genders.

- Recently diagnosed patients or not received systemic agents prior to the study for at least 3 months.

\section{Exclusion criteria:}

- Patients with psoriatic arthritis.

- Patients with arterial hypertension.

- Patients with diabetes mellitus.

- Patients with acute coronary syndrome or valvular heart disease.

- Patients with local or systemic infectious disease and inflammatory disorders.

- Patients who received any drugs that can possibly hinder the evaluation of endocan(angiotensin converting enzyme inhibitors and statins).

Complete history obtained and detailed dermatological examination was done for all patients. Psoriasis vulgaris was clinically diagnosed. Psoriasis was assessed by the same physician according to the (PASI) score[7]. We divided the patients depending on psoriasis severity to three categories (mild $<10$, moderate $10-20$, severe $>20$ ).

\section{Endocan measurements}

Three milliliters of venous blood samples were taken from every patients under complete aseptic precaution on sterile plane tube and were allowed to stand for 20 minutes at room temperature for coagulation, followed by centrifugation at speed of 2000-3000 r.p.m for 20 minutes. The separated serum was kept at $-20^{\circ} \mathrm{C}$ at the laboratory till the assay of endocan, which was measured by using an Enzyme Linked Immunosorbent assay(ELISA) kit provided by Sun Red company(Shanghai,China)(catalogue number:201-12-0026), that is depend on double-antibody sandwich technique to measure human endocan. For estimation of the results, the horizontal line represented the standard density and the vertical one for the optical density(OD) value, the graph paper showed the standard curve. We determined the corresponding density according to the sample OD value by the sample curve (The result is the sample density).

\section{Biochemical measurements}

The Westergren method was used to measure the erythrocyte sedimentation rate (ESR) with normal range $2-7 \mathrm{~mm} / \mathrm{h}$ for the first hour. We measured c-reactive protein (CRP) by using automated analyzer " Roche Cobas 8000-c702 ", supplied by (Roche Diagnostics, Germany) with normal range 1$5 \mathrm{mg} / \mathrm{ml}$.

\section{Statistical analysis}

Statistical Package of Social Science (SPSS), software version 24.0 (SPSS Inc., 2016) was used for data analysis. Mean \pm SD and Median(Range) used for continuous variables . Categorical variables were presented by the count and percentage. Normality was checked by Shapiro-Wilk test. The relation between variables were checked by pearson and spearman correlation analysis . The following tests were used ; Independentsamples t-test, one way analysis of variance (ANOVA) and post hoc Tukey test. A receiver operating characteristic (ROC) curve: used to detect the threshold value of optimal sensitivity and specificity of a test. Threshold for significance: $P$-value $<.05$ indicates significance , $P \leq .01$ highly significance, $P \leq .001$ very highly significance. $P \geq .05$ indicates non-significance.

\section{RESULTS}

This study included 22 patients with psoriasis vulgaris; 15 males $(68 \%)$ and 7 females $(32 \%)$. Patients' ages ranged from 18 to 65 years (mean $\pm \mathrm{SD}: 46.8 \pm 13.6$ ). Baseline demographic, disease characteristics, and laboratory data for the patients were presented in (Table 1).

The mean value of PASI score for psoriatic patients was 17.8 \pm 8.9 (range:336.8). According to psoriasis severity there were 6 patients with mild psoriasis , 8 moderate and 8 with severe disease. The mean value of serum endocan was $154 \pm 49.3 \mathrm{pg} / \mathrm{ml}$ (range 59-210).

A highly statistically significant positive correlation was detected between serum endocan and PASI score $\mathrm{P}=<0.001$ (Fig. 1). In contrast to, no statistically significant correlations were 
detected between serum endocan and ESR (first hour) and CRP $(\mathrm{P}=0.16, \quad \mathrm{P}=0.11$, respectively) (Table 2).

There were highly statistically significant differences in serum endocan levels among subgroups of severity. Serum endocan level had a statistically significant increase in moderate and severe psoriatic patients compared to mild psoriatic patients. $\mathrm{P} 1=0.006$ means statistically significant difference between mild and moderate psoriasis. $\mathrm{P} 2=<0.001$ means statistically significant difference between mild and severe psoriasis. No statistically significant difference in serum endocan level between moderate and severe psoriasis $(\mathrm{P} 3=0.25)$ (Table 3).

No statistically significant correlations were detected between serum endocan and patients' ages or disease duration $(\mathrm{P}=0.90$, $\mathrm{P}=0.75$, respectively).

Receiver operating characteristic (ROC) curve analysis detected that serum endocan can distinguish between patients with mild to moderate psoriasis vulgaris and patients with severe psoriasis vulgaris with an area under the curve (AUC) of 0.712 for serum endocan [(95\% confidence interval (CI): 00.482 to $0.883)$

The optimal sensitivity and specificity were $(80 \%$ and $66.7 \%$ respectively, at a cutoff expression value>176). This finding recommends that serum endocan is a potential prognostic biomarker in patients with psoriasis vulgaris. PASI score was dichotomized into severe vs mild or moderate (Fig. 2).

Table (1): Baseline, disease characteristics and laboratory findings in patients with psoriasis vulgaris

\begin{tabular}{|c|c|}
\hline Variables & \\
\hline Age(years) & \\
\hline Mean \pm SD & $46.8 \pm 13.6$ \\
\hline Median(Range) & $49(18-65)$ \\
\hline Sex, n, (\%) & \\
\hline Male & $15(68 \%)$ \\
\hline Female & $7(32 \%)$ \\
\hline Disease duration(n & \\
\hline Mean \pm SD & $47.1 \pm 40.5$ \\
\hline Median(Range) & $36(2-156)$ \\
\hline Family history, n, & \\
\hline Positive & $3(14 \%)$ \\
\hline Negative & $19(86 \%)$ \\
\hline Medical history, $\mathbf{n}$ & \\
\hline Positive & $0(0 \%)$ \\
\hline Negative & $22(100 \%)$ \\
\hline $\operatorname{ESR}(\mathbf{m m} / \mathbf{h})$ & \\
\hline Mean \pm SD & $21.6 \pm 15.7$ \\
\hline Median(Range) & $18.5(3-62)$ \\
\hline CRP (mg/ml) & \\
\hline Mean \pm SD & $6.4 \pm 6$ \\
\hline Median(Range) & $4.7(0.1-24)$ \\
\hline Endocan (pg/ml) & \\
\hline Mean \pm SD & $154 \pm 49.3$ \\
\hline Median(Range) & $178.5(59-210)$ \\
\hline
\end{tabular}

$\mathrm{SD}$, standard deviation; N, number; ESR, erythrocyte sedimentation rate; CRP, c-reactive protein. 
Table 2 : Correlation of endocan with clinical and laboratory findings in patients with psoriasis vulgaris

\begin{tabular}{|l|c|c|}
\hline Variables & & Endocan $(\mathrm{pg} / \mathrm{mL})$ \\
\hline ESR at first hour $(\mathbf{m m} / \mathbf{h})$ & $\boldsymbol{r}$ & .31 \\
& $\boldsymbol{P}$-value & .16 \\
\hline CRP $(\mathbf{m g} / \mathbf{m l})$ & $\boldsymbol{r}_{\boldsymbol{s}}$ & .35 \\
\hline (PASI) score & $\boldsymbol{P}$-value & .11 \\
\hline & $\boldsymbol{r}$ & $\mathbf{. 6 6}$ \\
\hline & $\boldsymbol{P}$-value & $\mathbf{. 0 0 1} * * *$ \\
\hline
\end{tabular}

PASI, Psoriasis Area and Severity Index; ESR, erythrocyte sedimentation rate; CRP, c-reactive protein; r, pearson correlation coefficient; rs, spearman's rho correlation coefficient,

Table 3: Association between severity by Psoriasis Area and Severity Index and endocan levels

\begin{tabular}{|c|c|c|c|c|c|}
\hline \multirow[t]{2}{*}{ Variables } & Mild & Moderate & Severe & \multirow[t]{2}{*}{ ANOVA } & \multirow[t]{2}{*}{$P$-value } \\
\hline & $n=6$ & $n=8$ & $n=8$ & & \\
\hline $\begin{array}{l}\text { Serum } \\
\text { endocan }(p g / m L)\end{array}$ & & & & \multirow[t]{4}{*}{$F=13.3$} & \multirow[t]{4}{*}{$<.001$} \\
\hline \multirow[t]{3}{*}{ mean $\pm \mathrm{SD}$} & $97.2 \pm 45.4$ & $161.5 \pm 37.8$ & $189.1 \pm 11.7$ & & \\
\hline & & $P 1=.006$ & $P 2<.001$ & & \\
\hline & & & $P 3=.25$ & & \\
\hline
\end{tabular}

$\mathrm{SD}$, standard deviation; F, analysis of variance(ANOVA)

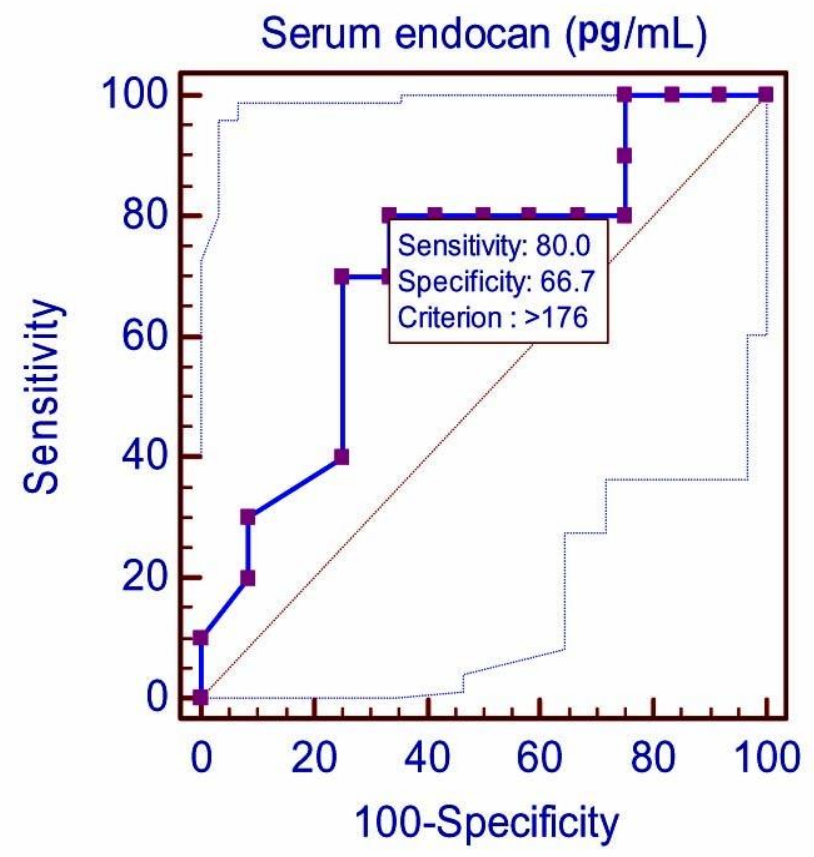

Figure 1: 


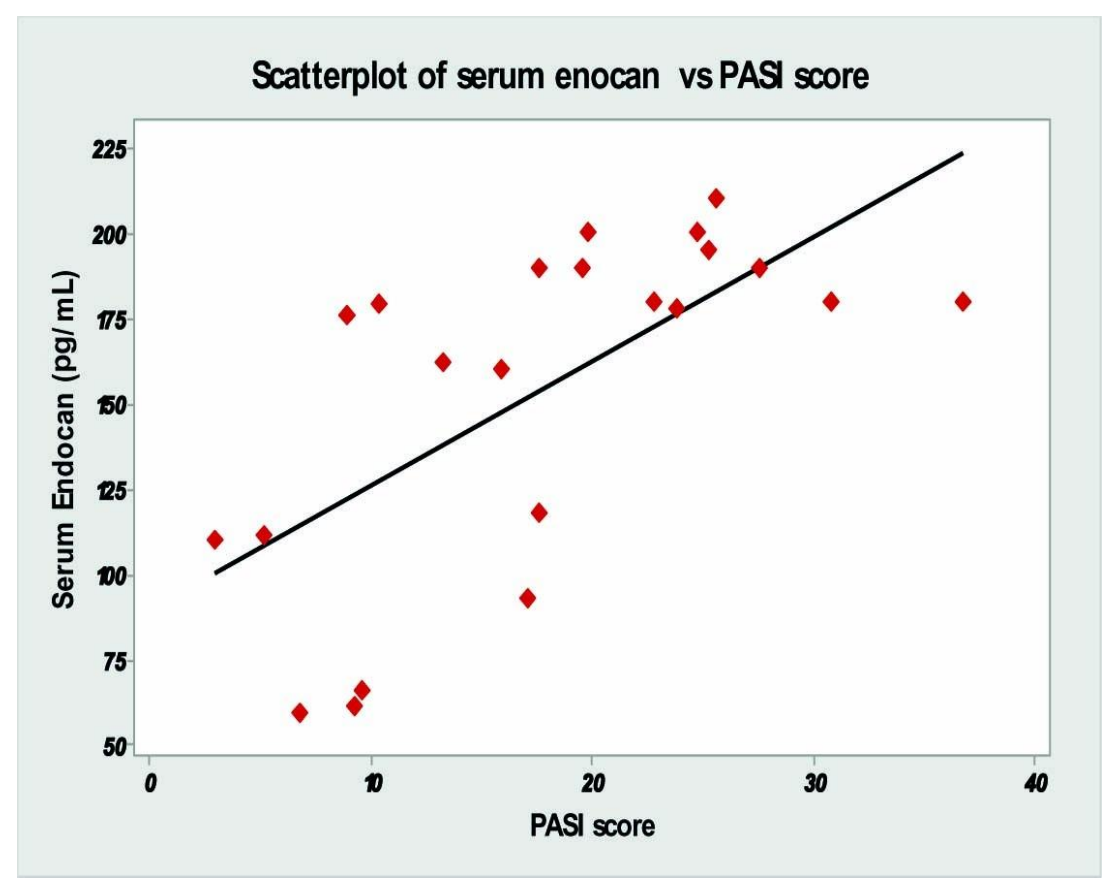

Figure 2:

\section{DISCUSSION}

Endocan is a soluble dermatan sulfate proteoglycan. Researches have shown that endocan is released by vascular endothelium, epithelium of distal tubules of the kidney, bronchi and submucosal glands of the lung[8].

Expression of endocan is under control of various cytokines and growth factors. Tumor necrosis factor-alpha(TNF $\alpha)$ and interleukin -1 beta(IL-1 $\beta)$ induce its expression in vitro, while interferon gamma(INF $\gamma$ ) inhibits upregulation of endocan induced by $\mathrm{TNF} \alpha[9]$. Angiogenesis depends mainly on vascular endothelial growth factor (VEGF-A) and (VEGF-C) which lead to upregulation of endocan expression[10].

Endocan has an important role in endothelium dependent pathological disorders by regulating cellular adhesion, migration and proliferation[11].

Our work aimed to evaluate the endocan role as a marker of psoriasis vulgaris severity.

In our study, the mean value of serum endocan in the patients was $154 \pm 49.3 \mathrm{pg} / \mathrm{ml}$ (range:59-210). A significant positive correlation was detected between PASI score and serum endocan level with statistically significant increase in serum level of endocan in moderate and severe psoriasis in comparison to mild psoriasis.

The serum endocan did not show significant correlations with ESR (first hour) or CRP ( $\mathrm{p}>0.05)$.

Balta et al.[12], detected a significant high serum levels of endocan in psoriasis vulgaris patients in comparison to control group $(\mathrm{p}<0.001)$ and positive correlation was detected between levels of endocan and disease activity (PASI) $(\mathrm{p}=0.009)$. Serum endocan did not statistically correlated with $\operatorname{ESR}(\mathrm{r}=0.181, \mathrm{p}=0.34)$.

Unlike our results they reported that endocan was correlated with CRP $(r=0.484$, $\mathrm{p}=0.008)$. We suggested that this difference referred to our utilization of conventional methods for CRP measurement rather the high sensitivity CRP (hs-CRP) utilized by Balta et al. Using this advanced technology can increase the accuracy of measurements especially for conditions with lower inflammatory burden. In addition to,the cutoff points for endocan were different between the 
two studies that may hinder the comparability of the findings.

Erek Toprak et al.[13],reported that the serum levels of endocan had no significant difference between patients and controls ( $p>0.05)$, serum endocan was significantly correlated with PASI score $(\mathrm{p}=0.006)$. The lower mean of PASI score $(11.34 \pm 7.29)$ of their patients and accordingly low systemic inflammation levels were possibly responsible for these insignificant results[14].

Supporting our findings, Gobrial et al.[15], demonstrated that endocan levels in serum and lesional skin showed statistically significant higher value in patients with psoriasis vulgaris and showed positive correlation with PASI score. They also reported that endocan levels in serum and tissue were significantly higher in patients with moderate to severe psoriasis than in those with mild disease.

In agreement with our findings, Sabry et al.[16], reported increased serum endocan levels in psoriatic patients than controls $(\mathrm{p}=0.0039)$ with positive significant correlation with PASI score $(\mathrm{p}=0.002)$.

Farag et al.[17], observed significant increase in serum endocan level in psoriasis vulgaris patients than control group $(\mathrm{p}<0.009)$ and the elevated level showed significant positive correlation with disease activity (PASI) score $\quad(\mathrm{p}<0.001)$ Recently, Elkamshoushi et al.[14], also demonstrated elevated serum levels of endocan that positively correlated with PASI score $(\mathrm{p}=0.002)$.

These findings encourage that endocan has an active role in the pathogenic mechanisms of psoriasis and contribute to disease severity.

Endocan can induce production of proinflammatory cytokines by endothelial cells and enhance the permeability of blood vessels. It has a possible role in cellular proliferation[18].

Endocan is involved in the regulation of leukocyte function associated antigen-1(LFA1)/intercellular adhesion molecule-1(ICAM1) pathway affecting both enrollment of circulating lymphocytes to the site of inflammation and leukocytes adhesion and activation depending on LFA-1[19].

Angiogenesis is considered as a crucial step in psoriasis. Endocan can promote the mitogenic and migratory activities of (VEGFA) a critical mediator in angiogenesis[20]

The small sample size and lack of control group were the main limitations of our work.

\section{CONCLUSIONS}

Serum endocan can be a promising marker in psoriasis vulgaris. It is valuable in prognosis and judgment of severity.

Endocan has a possible role in the pathogenesis of psoriasis, hence endocan represents an optimistic therapeutic target for psoriasis in the future.

\section{Declaration of interest :}

The authors report no conflicts of interest. The authors alone are responsible for the content and writing of the paper.

Funding information : None declared REFERENCES

1. Sarrazin S, Adam E, Lyon M, Depontieu F, Motte V, Landolfi C, et al. Endocan or endothelial cell specific molecule1(ESM-1): a potential novel endothelial cell marker and a new target for cancer therapy. Biochim Biophys Acta 2006; 1765:25-37.

2. Bèchard $D$, Gentina $T$, Delehedde $M$, Scherpereel A, Lyon M, Aumercier M, et al. Endocan is a novel chondroitin sulfate/dermatan sulfate proteoglycan that promotes hepatocyte growth factor/ scatter factor mitogenic activity. J Biol Chem 2001;276:48341-48349.

3. Kali A, Shetty KS. Endocan : a novel circulating proteoglycan. Indian $\mathbf{J}$ Pharmacol 2014; 46:579-583.

4. Sabat R, Philipp S, Höflich C, Kreutzer $\mathrm{S}$, Wallace $\mathrm{E}$, Asadullah $\mathrm{K}$, et al. Immunopathogenesis of psoriasis. Exp Dermatol 2007; 16:779-798.

5. Krueger JG, Bowcock A. Psoriasis pathophysiology: Current concepts of pathogenesis. Ann Rheum Dis 2006; 64(suppl.2):ii30-36.

6. Ghazizadeh R, Shimizu H, Tosa M, Ghazizadeh M. Pathogenic mechanisms shared between psoriasis and 
cardiovascular disease. Int J Med Sci 2010;7:284-289.

7. Faria JR, Aarão AR, Jimenez LM, Silva $\mathrm{OH}$, Avelleira JC. Inter-rater concordance study of the PASI (Psoriasis Area and Severity Index). An Bras Dermatol 2010; 85:625-629.

8. Zhang SM, Zuo L, Zhou Q, Gui SY, Shi $\mathrm{R}, \mathrm{Wu} \mathrm{Q}$, et al. Expression and distribution of endocan in human tissues. Biotech Histochem 2012; 87:172-178.

9. Delehedde M, Devenyns L, Maurage CA, Vivès RR. Endocan in cancers: a lesson from a circulating dermatan sulfate proteoglycan. Int J Cell Biol 2013, 2013:705027.

10. Shin JW, Huggenberger R, Detmar M. Transcriptional profiling of VEGF-A and VEGF-C target genes in lymphatic endothelium reveals endothelial-specific molecule-1 as a novel mediator of lymphangiogenesis. Blood 2008; 112:2318-2326.

11. Scherpereel A, Depontieu F, Grigoriu B, Cavestri B, Tsicopoulos A, Gentina T, et al. Endocan, a new endothelial marker in human sepsis. Crit Care Med 2006;34:532-537.

12. Balta I, Balta S, Demirkol S, Mikhailidis DP, Celik T, Akhan M, et al. Elevated serum levels of endocan in patients with psoriasis vulgaris: correlations with cardiovascular risk and activity of disease. Br J Dermatol 2013; 169:10661070.

13. Erek Toprak A, Ozlu E, Uzuncakmak TK, Yalcinkaya E, Sogut S, Karadag AS. Neutrophil/lymphocyte ratio, serum endocan and nesfatin-1 levels in patients with psoriasis vulgaris undergoing phototherapy treatment. Med Sci Monit 2016; 22:1232-1237.

14. Elkamshoushi AM, Omar SS, El Abd AM, Hassan SZ, Sultan EA, Abd Elkawy E. Subclinical atherosclerosis in psoriatic disease: relation to endocan, TNF- $\alpha$, age of onset, and body fat. Int $\mathrm{J}$ Dermatol 2019; 58(4):456-464.

15. Gobrial CK, Assad MK, Seifeldin NS, Elskhawy YN, Abdelaziz DM. Endocan level in serum and lesional skin of psoriasis vulgaris patients. JEWDS 2017; 14:179-184.

16. Sabry HH, Sabry JH, Daifalla AEH, Akl EM, Hamed AM, Torky AAA. Serum markers for asymptomatic atherosclerosis in Egyptian psoriatic patients: study controlled by Doppler estimation of carotid intima-media thickness. Vasc Health Risk Manag 2018;14:145-152.

17. Farag AGA, Elshayeb EI, Habib MS, Ibrahem RA, Qutubuddin M, Mahfouz RZ. Endocan: a reliable biomarker for renal impairment in psoriasis vulgaris patients. EJPMR 2018; 5(6):120-129.

18. Balta S, Mikhailidis DP, Demirkol S, Ozturk C, Celik T, Lyisoy A. Endocan: a novel inflammatory indicator in cardiovascular disease. Atherosclerosis 2015; 243:339-343.

19. Bèchard D, Scherpereel A, Hammad H, Gentina T, Tsicopoulous A, Aumercier $\mathrm{M}$, et al. Human endothelial-cell specific molecule-1 binds directly to the integrin CD11a/CD18(LFA-1) and blocks binding to intercellular adhesion molecule-1. J Immunol 2001; 167:3099-3106.

20. Gelfand JM, Yeung H. Metabolic syndrome in patients with psoriatic disease. J Rheumatol Suppl 2012; 89:2428.

To Cite This Article Elgarib I, Khalifa N, Elasser O, ElGhareeb M, Hosny D . Serum Endocan Levels in Patients with Psoriasis Vulgaris as a Marker of Disease Severity . Zumj May. 2020(26) No.3,517-523. DOI: 10.21608/zumj.2019.13587.1252 